\title{
A locally compact quantum group of triangular matrices.
}

\author{
Pierre Fima* and Leonid Vainerman ${ }^{\dagger}$ \\ Dedicated to Professor M.L. Gorbachuk on the occasion of his \\ 70-th anniversary.
}

\begin{abstract}
We construct a one parameter deformation of the group of $2 \times 2$ upper triangular matrices with determinant 1 using the twisting construction. An interesting feature of this new example of a locally compact quantum group is that the Haar measure is deformed in a non-trivial way. Also, we give a complete description of the dual $C^{*}$-algebra and the dual comultiplication.
\end{abstract}

\section{Introduction}

In 3, 14, M. Enock and the second author proposed a systematic approach to the construction of non-trivial Kac algebras by twisting. To illustrate it, consider a cocommutative Kac algebra structure on the group von Neumann algebra $M=\mathcal{L}(G)$ of a non commutative locally compact (l.c.) group $G$ with comultiplication $\Delta\left(\lambda_{g}\right)=\lambda_{g} \otimes \lambda_{g}$ (here $\lambda_{g}$ is the left translation by $\left.g \in G\right)$. Let us define on $M$ another, "twisted", comultiplication $\Delta_{\Omega}(\cdot)=\Omega \Delta(\cdot) \Omega^{*}$, where $\Omega$ is a unitary from $M \otimes M$ verifying certain 2-cocycle condition, and construct in this way new, non cocommutative, Kac algebra structure on $M$. In order to find such an $\Omega$, let us, following to M. Rieffel [10] and M. Landstad 8], take an inclusion $\alpha: L^{\infty}(\hat{K}) \rightarrow M$, where $\hat{K}$ is the dual to some abelian subgroup $K$ of $G$ such that $\left.\delta\right|_{K}=1$, where $\delta(\cdot)$ is the module of $G$. Then, one lifts a usual 2-cocycle $\Psi$ of $\hat{K}: \Omega=(\alpha \otimes \alpha) \Psi$. The main result of [3], [14] is that the integral by the Haar measure of $G$ gives also the Haar measure of the deformed object. Recently P. Kasprzak studied the deformation of l.c. groups by twisting in [5], and also in this case the Haar measure was not deformed.

In [4, the authors extended the twisting construction in order to cover the case of non-trivial deformation of the Haar measure. The aim of the present paper is to illustrate this construction on a concrete example and to compute

\footnotetext{
* Laboratoire de Mathématiques, Université de Franche-Comté, 16 route de Gray, 25030 Besancon Cedex, France. E-mail: fima@math.unicaen.fr

${ }^{\dagger}$ Laboratoire de Mathématiques Nicolas Oresme, Université de Caen, B.P. 5186, 14032 Caen Cedex, France. E-mail: vainerman@math.unicaen.fr
} 
explicitly all the ingredients of the twisted quantum group including the dual $C^{*}$ algebra and the dual comultiplication. We twist the group von Neumann algebra $\mathcal{L}(G)$ of the group $G$ of $2 \times 2$ upper triangular matrices with determinant 1 using the abelian subgroup $K=\mathbb{C}^{*}$ of diagonal matrices of $G$ and a one parameter family of bicharacters on $K$. In this case, the subgroup $K$ is not included in the kernel of the modular function of $G$, this is why the Haar measure is deformed. We compute the new Haar measure and show that the dual $C^{*}$ algebra is generated by 2 normal operators $\hat{\alpha}$ and $\hat{\beta}$ such that

$$
\hat{\alpha} \hat{\beta}=\hat{\beta} \hat{\alpha} \quad \hat{\alpha} \hat{\beta}^{*}=q \hat{\beta}^{*} \hat{\alpha},
$$

where $q>0$. Moreover, the comultiplication $\hat{\Delta}$ is given by

$$
\hat{\Delta}_{t}(\hat{\alpha})=\hat{\alpha} \otimes \hat{\alpha}, \hat{\Delta}_{t}(\hat{\beta})=\hat{\alpha} \otimes \hat{\beta} \dot{+} \hat{\beta} \otimes \hat{\alpha}^{-1},
$$

where $\dot{+}$ means the closure of the sum of two operators.

This paper in organized as follows. In Section 2 we recall some basic definitions and results. In Section 3 we present in detail our example computing all the ingredients associated. This example is inspired by [5, but an important difference is that in the present example the Haar measure is deformed in a non trivial way. Finally, we collect some useful results in the Appendix.

\section{Preliminaries}

\section{$2.1 \quad$ Notations}

Let $B(H)$ be the algebra of all bounded linear operators on a Hilbert space $H, \otimes$ the tensor product of Hilbert spaces, von Neumann algebras or minimal tensor product of $C^{*}$-algebras, and $\Sigma$ (resp., $\sigma$ ) the flip map on it. If $H, K$ and $L$ are Hilbert spaces and $X \in B(H \otimes L)$ (resp., $X \in B(H \otimes K), X \in B(K \otimes L)$ ), we denote by $X_{13}$ (resp., $\left.X_{12}, X_{23}\right)$ the operator $\left(1 \otimes \Sigma^{*}\right)(X \otimes 1)(1 \otimes \Sigma)$ (resp., $X \otimes 1,1 \otimes X$ ) defined on $H \otimes K \otimes L$. For any subset $X$ of a Banach space $E$, we denote by $\langle X\rangle$ the vector space generated by $X$ and $[X]$ the closed vector space generated by $X$. All l.c. groups considered in this paper are supposed to be second countable, all Hilbert spaces are separable and all von Neumann algebras have separable preduals.

Given a normal semi-finite faithful (n.s.f.) weight $\theta$ on a von Neumann algebra $M$ (see [12]), we denote: $\mathcal{M}_{\theta}^{+}=\left\{x \in M^{+} \mid \theta(x)<+\infty\right\}, \mathcal{N}_{\theta}=\{x \in$ $\left.M \mid x^{*} x \in M_{\theta}^{+}\right\}$, and $\mathcal{M}_{\theta}=\left\langle\mathcal{M}_{\theta}^{+}\right\rangle$.

When $A$ and $B$ are $C^{*}$-algebras, we denote by $\mathrm{M}(A)$ the algebra of the multipliers of $A$ and by $\operatorname{Mor}(A, B)$ the set of the morphisms from $A$ to $B$.

\section{$2.2 \quad G$-products and their deformation}

For the notions of an action of a l.c. group $G$ on a $C^{*}$-algebra $A$, a $C^{*}$ dynamical system $(A, G, \alpha)$, a crossed product $G_{\alpha} \ltimes A$ of $A$ by $G$ see [9]. The crossed product has the following universal property: 
For any $C^{*}$-covariant representation $(\pi, u, B)$ of $(A, G, \alpha)$ (here $B$ is a $C^{*}$ algebra, $\pi: A \rightarrow B$ a morphism, $u$ is a group morphism from $G$ to the unitaries of $M(B)$, continuous for the strict topology), there is a unique morphism $\rho \in$ $\operatorname{Mor}\left(G_{\alpha} \ltimes A, B\right)$ such that

$$
\rho\left(\lambda_{t}\right)=u_{t}, \quad \rho\left(\pi_{\alpha}(x)\right)=\pi(x) \quad \forall t \in G, x \in A .
$$

Definition 1 Let $G$ be a l.c. abelian group, $B$ a $C^{*}$-algebra, $\lambda$ a morphism from $G$ to the unitary group of $M(B)$, continuous in the strict topology of $M(B)$, and $\theta$ a continuous action of $\hat{G}$ on $B$. The triplet $(B, \lambda, \theta)$ is called a $G$-product if $\theta_{\gamma}\left(\lambda_{g}\right)=\overline{\langle\gamma, g\rangle} \lambda_{g}$ for all $\gamma \in \hat{G}, g \in G$.

The unitary representation $\lambda: G \rightarrow \mathrm{M}(B)$ generates a morphism :

$$
\lambda \in \operatorname{Mor}\left(C^{*}(G), B\right) .
$$

Identifying $C^{*}(G)$ with $C_{0}(\hat{G})$, one gets a morphism $\lambda \in \operatorname{Mor}\left(C_{0}(\hat{G}), B\right)$ which is defined in a unique way by its values on the characters

$$
u_{g}=(\gamma \mapsto\langle\gamma, g\rangle) \in C_{b}(\hat{G}): \lambda\left(u_{g}\right)=\lambda_{g}, \quad \text { for all } g \in G
$$

One can check that $\lambda$ is injective.

The action $\theta$ is done by: $\theta_{\gamma}\left(\lambda\left(u_{g}\right)\right)=\theta_{\gamma}\left(\lambda_{g}\right)=\overline{\langle\gamma, g\rangle} \lambda_{g}=\lambda\left(u_{g}(.-\gamma)\right)$. Since the $u_{g}$ generate $C_{b}(\hat{G})$, one deduces that:

$$
\theta_{\gamma}(\lambda(f))=\lambda(f(.-\gamma)), \quad \text { for all } f \in C_{b}(\hat{G})
$$

The following definition is equivalent to the original definition by Landstad [8] (see [5]):

Definition 2 Let $(B, \lambda, \theta)$ be a $G$-poduct and $x \in M(B)$. One says that $x$ verifies the Landstad conditions if

$$
\begin{cases}(i) & \theta_{\gamma}(x)=x, \quad \text { for any } \gamma \in \hat{G} \\ (\text { ii }) & \text { the application } g \mapsto \lambda_{g} x \lambda_{g}^{*} \text { is continuous } \\ (\text { iii }) & \lambda(f) x \lambda(g) \in B, \quad \text { for any } f, g \in C_{0}(\hat{G})\end{cases}
$$

The set $A \in \mathrm{M}(B)$ verifying these conditions is a $C^{*}$-algebra called the Landstad algebra of the $G$-product $(B, \lambda, \theta)$. Definition 2 implies that if $a \in A$, then $\lambda_{g} a \lambda_{g}^{*} \in A$ and the map $g \mapsto \lambda_{g} a \lambda_{g}^{*}$ is continuous. One gets then an action of $G$ on $A$.

One can show that the inclusion $A \rightarrow \mathrm{M}(B)$ is a morphism of $C^{*}$-algebras, so $\mathrm{M}(A)$ can be also included into $\mathrm{M}(B)$. If $x \in \mathrm{M}(B)$, then $x \in \mathrm{M}(A)$ if and only if

$$
\begin{cases}(i) & \theta_{\gamma}(x)=x, \text { for all } \gamma \in \hat{G} \\ (\text { ii }) & \text { for all } a \in A, \text { the application } g \mapsto \lambda_{g} x \lambda_{g}^{*} a \text { is continuous. }\end{cases}
$$

Let us note that two first conditions of (11) imply (2). 
The notions of $G$-product and crossed product are closely related. Indeed, if $(A, G, \alpha)$ is a $C^{*}$-dynamical system with $G$ abelian, let $B=G_{\alpha} \ltimes A$ be the crossed product and $\lambda$ the canonical morphism from $G$ into the unitary group of $\mathrm{M}(B)$, continuous in the strict topology, and $\pi \in \operatorname{Mor}(A, B)$ the canonical morphism of $C^{*}$-algebras. For $f \in \mathcal{K}(G, A)$ and $\gamma \in \hat{G}$, one defines $\left(\theta_{\gamma} f\right)(t)=$ $\overline{\langle\gamma, t\rangle} f(t)$. One shows that $\theta_{\gamma}$ can be extended to the automorphisms of $B$ in such a way that $(B, \hat{G}, \theta)$ would be a $C^{*}$-dynamical system. Moreover, $(B, \lambda, \theta)$ is a $G$-product and the associated Landstad algebra is $\pi(A)$. $\theta$ is called the dual action. Conversely, if $(B, \lambda, \theta)$ is a $G$-product, then one shows that there exists a $C^{*}$-dynamical system $(A, G, \alpha)$ such that $B=G_{\alpha} \ltimes A$. It is unique (up to a covariant isomorphism), $A$ is the Landstad algebra of $(B, \lambda, \theta)$ and $\alpha$ is the action of $G$ on $A$ given by $\alpha_{t}(x)=\lambda_{t} x \lambda_{t}^{*}$.

Lemma 1 [5] Let $(B, \lambda, \theta)$ be a $G$-product and $V \subset A$ be a vector subspace of the Landstad algebra such that:

- $\lambda_{g} V \lambda_{g}^{*} \subset V$, for any $g \in G$,

- $\lambda\left(C_{0}(\hat{G})\right) V \lambda\left(C_{0}(\hat{G})\right)$ is dense in $B$.

Then $V$ is dense in $A$.

Let $(B, \lambda, \theta)$ be a $G$-product, $A$ its Landstad algebra, and $\Psi$ a continuous bicharacter on $\hat{G}$. For $\gamma \in \hat{G}$, the function on $\hat{G}$ defined by $\Psi_{\gamma}(\omega)=\Psi(\omega, \gamma)$ generates a family of unitaries $\lambda\left(\Psi_{\gamma}\right) \in \mathrm{M}(B)$. The bicharacter condition implies:

$$
\theta_{\gamma}\left(U_{\gamma_{2}}\right)=\lambda\left(\Psi_{\gamma_{2}}\left(.-\gamma_{1}\right)\right)=\overline{\Psi\left(\gamma_{1}, \gamma_{2}\right)} U_{\gamma_{2}}, \quad \forall \gamma_{1}, \gamma_{2} \in \hat{G} .
$$

One gets then a new action $\theta^{\Psi}$ of $\hat{G}$ on $B$ :

$$
\theta_{\gamma}^{\Psi}(x)=U_{\gamma} \theta(x) U_{\gamma}^{*}
$$

Note that, by commutativity of $G$, one has:

$$
\theta_{\gamma}^{\Psi}\left(\lambda_{g}\right)=U_{\gamma} \theta\left(\lambda_{g}\right) U_{\gamma}^{*}=\overline{\langle\gamma, g\rangle} \lambda_{g}, \quad \forall \gamma \in \hat{G}, g \in G .
$$

The triplet $\left(B, \lambda, \theta^{\Psi}\right)$ is then a $G$-product, called a deformed $G$-product.

\subsection{Locally compact quantum groups [6], [7]}

A pair $(M, \Delta)$ is called a (von Neumann algebraic) l.c. quantum group when

- $M$ is a von Neumann algebra and $\Delta: M \rightarrow M \otimes M$ is a normal and unital $*$-homomorphism which is coassociative: $(\Delta \otimes$ id $) \Delta=($ id $\otimes \Delta) \Delta$ (i.e., $(M, \Delta)$ is a Hopf-von Neumann algebra).

- There exist n.s.f. weights $\varphi$ and $\psi$ on $M$ such that

$-\varphi$ is left invariant in the sense that $\varphi((\omega \otimes$ id $) \Delta(x))=\varphi(x) \omega(1)$ for all $x \in \mathcal{M}_{\varphi}^{+}$and $\omega \in M_{*}^{+}$, 
- $\psi$ is right invariant in the sense that $\psi((\operatorname{id} \otimes \omega) \Delta(x))=\psi(x) \omega(1)$ for all $x \in \mathcal{M}_{\psi}^{+}$and $\omega \in M_{*}^{+}$.

Left and right invariant weights are unique up to a positive scalar.

Let us represent $M$ on the GNS Hilbert space of $\varphi$ and define a unitary $W$ on $H \otimes H$ by

$$
W^{*}(\Lambda(a) \otimes \Lambda(b))=(\Lambda \otimes \Lambda)(\Delta(b)(a \otimes 1)), \quad \text { for all } a, b \in N_{\phi} .
$$

Here, $\Lambda$ denotes the canonical GNS-map for $\varphi, \Lambda \otimes \Lambda$ the similar map for $\varphi \otimes \varphi$. One proves that $W$ satisfies the pentagonal equation: $W_{12} W_{13} W_{23}=W_{23} W_{12}$, and we say that $W$ is a multiplicative unitary. The von Neumann algebra $M$ and the comultiplication on it can be given in terms of $W$ respectively as

$$
M=\left\{(\operatorname{id} \otimes \omega)(W) \mid \omega \in B(H)_{*}\right\}^{-\sigma-s t r o n g *}
$$

and $\Delta(x)=W^{*}(1 \otimes x) W$, for all $x \in M$. Next, the l.c. quantum group $(M, \Delta)$ has an antipode $S$, which is the unique $\sigma$-strongly* closed linear map from $M$ to $M$ satisfying (id $\otimes \omega)(W) \in \mathcal{D}(S)$ for all $\omega \in B(H)_{*}$ and $S($ id $\otimes \omega)(W)=$ $($ id $\otimes \omega)\left(W^{*}\right)$ and such that the elements $($ id $\otimes \omega)(W)$ form a $\sigma$-strong* core for $S$. $S$ has a polar decomposition $S=R \tau_{-i / 2}$, where $R$ (the unitary antipode) is an anti-automorphism of $M$ and $\tau_{t}$ (the scaling group of $(M, \Delta)$ ) is a strongly continuous one-parameter group of automorphisms of $M$. We have $\sigma(R \otimes R) \Delta=$ $\Delta R$, so $\varphi R$ is a right invariant weight on $(M, \Delta)$ and we take $\psi:=\varphi R$.

Let $\sigma_{t}$ be the modular automorphism group of $\varphi$. There exist a number $\nu>0$, called the scaling constant, such that $\psi \sigma_{t}=\nu^{-t} \psi$ for all $t \in \mathbb{R}$. Hence (see [13]), there is a unique positive, self-adjoint operator $\delta_{M}$ affiliated to $M$, such that $\sigma_{t}\left(\delta_{M}\right)=\nu^{t} \delta_{M}$ for all $t \in \mathbb{R}$ and $\psi=\varphi_{\delta_{M}}$. It is called the modular element of $(M, \Delta)$. If $\delta_{M}=1$ we call $(M, \Delta)$ unimodular. The scaling constant can be characterized as well by the relative invariance $\varphi \tau_{t}=\nu^{-t} \varphi$.

For the dual l.c. quantum group $(\hat{M}, \hat{\Delta})$ we have :

$$
\hat{M}=\left\{(\omega \otimes \mathrm{id})(W) \mid \omega \in B(H)_{*}\right\}^{-\sigma-\text { strong* }}
$$

and $\hat{\Delta}(x)=\Sigma W(x \otimes 1) W^{*} \Sigma$ for all $x \in \hat{M}$. A left invariant n.s.f. weight $\hat{\varphi}$ on $\hat{M}$ can be constructed explicitly and the associated multiplicative unitary is $\hat{W}=\Sigma W^{*} \Sigma$.

Since $(\hat{M}, \hat{\Delta})$ is again a l.c. quantum group, let us denote its antipode by $\hat{S}$, its unitary antipode by $\hat{R}$ and its scaling group by $\hat{\tau}_{t}$. Then we can construct the dual of $(\hat{M}, \hat{\Delta})$, starting from the left invariant weight $\hat{\varphi}$. The bidual l.c. quantum group $(\hat{\hat{M}}, \hat{\hat{\Delta}})$ is isomorphic to $(M, \Delta)$.

$M$ is commutative if and only if $(M, \Delta)$ is generated by a usual l.c. group $G: M=L^{\infty}(G),\left(\Delta_{G} f\right)(g, h)=f(g h),\left(S_{G} f\right)(g)=f\left(g^{-1}\right), \varphi_{G}(f)=\int f(g) d g$, where $f \in L^{\infty}(G), g, h \in G$ and we integrate with respect to the left Haar measure $d g$ on $G$. Then $\psi_{G}$ is given by $\psi_{G}(f)=\int f\left(g^{-1}\right) d g$ and $\delta_{M}$ by the strictly positive function $g \mapsto \delta_{G}(g)^{-1}$.

$L^{\infty}(G)$ acts on $H=L^{2}(G)$ by multiplication and $\left(W_{G} \xi\right)(g, h)=\xi\left(g, g^{-1} h\right)$, for all $\xi \in H \otimes H=L^{2}(G \times G)$. Then $\hat{M}=\mathcal{L}(G)$ is the group von Neumann 
algebra generated by the left translations $\left(\lambda_{g}\right)_{g \in G}$ of $G$ and $\hat{\Delta}_{G}\left(\lambda_{g}\right)=\lambda_{g} \otimes \lambda_{g}$. Clearly, $\hat{\Delta}_{G}^{o p}:=\sigma \circ \hat{\Delta}_{G}=\hat{\Delta}_{G}$, so $\hat{\Delta}_{G}$ is cocommutative.

$(M, \Delta)$ is a Kac algebra (see [2]) if $\tau_{t}=\mathrm{id}$, for all $t$, and $\delta_{M}$ is affiliated with the center of $M$. In particular, this is the case when $M=L^{\infty}(G)$ or $M=\mathcal{L}(G)$.

We can also define the $C^{*}$-algebra of continuous functions vanishing at infinity on $(M, \Delta)$ by

$$
A=\left[(\operatorname{id} \otimes \omega)(W) \mid \omega \in \mathcal{B}(H)_{*}\right]
$$

and the reduced $C^{*}$-algebra (or dual $C^{*}$-algebra) of $(M, \Delta)$ by

$$
\hat{A}=\left[(\omega \otimes \mathrm{id})(W) \mid \omega \in \mathcal{B}(H)_{*}\right] .
$$

In the group case we have $A=C_{0}(G)$ and $\hat{A}=C_{r}(G)$. Moreover, we have $\Delta \in \operatorname{Mor}(A, A \otimes A)$ and $\hat{\Delta} \in \operatorname{Mor}(\hat{A}, \hat{A} \otimes \hat{A})$.

A l.c. quantum group is called compact if $\varphi\left(1_{M}\right)<\infty$ and discrete if its dual is compact.

\subsection{Twisting of locally compact quantum groups [4]}

Let $(M, \Delta)$ be a locally compact quantum group and $\Omega$ a unitary in $M \otimes M$. We say that $\Omega$ is a 2 -cocycle on $(M, \Delta)$ if

$$
(\Omega \otimes 1)(\Delta \otimes \mathrm{id})(\Omega)=(1 \otimes \Omega)(\mathrm{id} \otimes \Delta)(\Omega) .
$$

As an example we can consider $M=L^{\infty}(G)$, where $G$ is a l.c. group, with $\Delta_{G}$ as above, and $\Omega=\Psi(\cdot, \cdot) \in L^{\infty}(G \times G)$ a usual 2-cocycle on $G$, i.e., a mesurable function with values in the unit circle $\mathbb{T} \subset \mathbb{C}$ verifying

$$
\Psi\left(s_{1}, s_{2}\right) \Psi\left(s_{1} s_{2}, s_{3}\right)=\Psi\left(s_{2}, s_{3}\right) \Psi\left(s_{1}, s_{2} s_{3}\right) \text {, for almost all } s_{1}, s_{2}, s_{3} \in G .
$$

This is the case for any measurable bicharacter on $G$.

When $\Omega$ is a 2-cocycle on $(M, \Delta)$, one can check that $\Delta_{\Omega}(\cdot)=\Omega \Delta(\cdot) \Omega^{*}$ is a new coassociative comultiplication on $M$. If $(M, \Delta)$ is discrete and $\Omega$ is any 2-cocycle on it, then $\left(M, \Delta_{\Omega}\right)$ is again a l.c. quantum group (see [1, finitedimensional case was treated in [14]). In the general case, one can proceed as follows. Let $\alpha:\left(L^{\infty}(G), \Delta_{G}\right) \rightarrow(M, \Delta)$ be an inclusion of Hopf-von Neumann algebras, i.e., a faithful unital normal ${ }^{*}$-homomorphism such that $(\alpha \otimes \alpha) \circ \Delta_{G}=$ $\Delta \circ \alpha$. Such an inclusion allows to construct a 2-cocycle of $(M, \Delta)$ by lifting a usual 2-cocycle of $G: \Omega=(\alpha \otimes \alpha) \Psi$. It is shown in 3 that if the image of $\alpha$ is included into the centralizer of the left invariant weight $\varphi$, then $\varphi$ is also left invariant for the new comultiplication $\Delta_{\Omega}$.

In particular, let $G$ be a non commutative l.c. group and $K$ a closed abelian subgroup of $G$. By Theorem 6 of [11, there exists a faithful unital normal *-homomorphism $\hat{\alpha}: \mathcal{L}(K) \rightarrow \mathcal{L}(G)$ such that

$$
\hat{\alpha}\left(\lambda_{g}^{K}\right)=\lambda_{g}, \quad \text { for all } g \in K, \quad \text { and } \quad \hat{\Delta} \circ \hat{\alpha}=(\hat{\alpha} \otimes \hat{\alpha}) \circ \hat{\Delta}_{K},
$$


where $\lambda^{K}$ and $\lambda$ are the left regular representation of $K$ and $G$ respectively, and $\hat{\Delta}_{K}$ and $\hat{\Delta}$ are the comultiplications on $\mathcal{L}(K)$ and $\mathcal{L}(G)$ repectively. The composition of $\hat{\alpha}$ with the canonical isomorphism $L^{\infty}(\hat{K}) \simeq \mathcal{L}(K)$ given by the Fourier tranformation, is a faithful unital normal *-homomorphism $\alpha$ : $L^{\infty}(\hat{K}) \rightarrow \mathcal{L}(G)$ such that $\Delta \circ \alpha=(\alpha \otimes \alpha) \circ \Delta_{\hat{K}}$, where $\Delta_{\hat{K}}$ is the comultiplication on $L^{\infty}(\hat{K})$. The left invariant weight on $\mathcal{L}(G)$ is the Plancherel weight for which

$$
\sigma_{t}(x)=\delta_{G}^{i t} x \delta_{G}^{-i t}, \quad \text { for all } x \in \mathcal{L}(G),
$$

where $\delta_{G}$ is the modular function of $G$. Thus, $\sigma_{t}\left(\lambda_{g}\right)=\delta_{G}^{i t}(g) \lambda_{g}$ or

$$
\sigma_{t} \circ \alpha\left(u_{g}\right)=\alpha\left(u_{g}\left(\cdot-\gamma_{t}\right)\right)
$$

where $u_{g}(\gamma)=\langle\gamma, g\rangle, g \in G, \gamma \in \hat{G}, \gamma_{t}$ is the character $K$ defined by $\left\langle\gamma_{t}, g\right\rangle=$ $\delta_{G}^{-i t}(g)$. By linearity and density we obtain:

$$
\sigma_{t} \circ \alpha(F)=\alpha\left(F\left(\cdot-\gamma_{t}\right)\right), \quad \text { for all } F \in L^{\infty}(\hat{K}) .
$$

This is why we do the following assumptions. Let $(M, \Delta)$ be a l.c. quantum group, $G$ an abelian l.c. group and $\alpha:\left(L^{\infty}(G), \Delta_{G}\right) \rightarrow(M, \Delta)$ an inclusion of Hopf-von Neumann algebras. Let $\varphi$ be the left invariant weight, $\sigma_{t}$ its modular group, $S$ the antipode, $R$ the unitary antipode, $\tau_{t}$ the scaling group. Let $\psi=$ $\varphi \circ R$ be the right invariant weight and $\sigma_{t}^{\prime}$ its modular group. Also we denote by $\delta$ the modular element of $(M, \Delta)$. Suppose that there exists a continuous group homomorphism $t \mapsto \gamma_{t}$ from $\mathbb{R}$ to $G$ such that

$$
\sigma_{t} \circ \alpha(F)=\alpha\left(F\left(\cdot-\gamma_{t}\right)\right), \quad \text { for all } F \in L^{\infty}(G) .
$$

Let $\Psi$ be a continuous bicharacter on $G$. Notice that $(t, s) \mapsto \Psi\left(\gamma_{t}, \gamma_{s}\right)$ is a continuous bicharacter on $\mathbb{R}$, so there exists $\lambda>0$ such that $\Psi\left(\gamma_{t}, \gamma_{s}\right)=\lambda^{i s t}$. We define:

$$
u_{t}=\lambda^{i \frac{t^{2}}{2}} \alpha\left(\Psi\left(.,-\gamma_{t}\right)\right) \quad \text { and } \quad v_{t}=\lambda^{i \frac{t^{2}}{2}} \alpha\left(\Psi\left(-\gamma_{t}, .\right)\right) .
$$

The 2-cocycle equation implies that $u_{t}$ is a $\sigma_{t}$-cocyle and $v_{t}$ is a $\sigma_{t}^{\prime}$-cocycle. The Connes' Theorem gives two n.s.f. weights on $M, \varphi_{\Omega}$ and $\psi_{\Omega}$, such that

$$
u_{t}=\left[D \varphi_{\Omega}: D \varphi\right]_{t} \quad \text { and } \quad v_{t}=\left[D \psi_{\Omega}: D \psi\right]_{t} .
$$

The main result of 4 is as follows:

Theorem $1\left(M, \Delta_{\Omega}\right)$ is a l.c. quantum group with left and right invariant weight $\varphi_{\Omega}$ and $\psi_{\Omega}$ respectively. Moreover, denoting by a subscript or a superscript $\Omega$ the objects associated with $\left(M, \Delta_{\Omega}\right)$ one has:

- $\tau_{t}^{\Omega}=\tau_{t}$,

- $\nu_{\Omega}=\nu$ and $\delta_{\Omega}=\delta A^{-1} B$, 
- $\mathcal{D}\left(S_{\Omega}\right)=\mathcal{D}(S)$ and, for all $x \in \mathcal{D}(S), S_{\Omega}(x)=u S(x) u^{*}$.

Remark that, because $\Psi$ is a bicharacter on $G, t \mapsto \alpha\left(\Psi\left(.,-\gamma_{t}\right)\right)$ is a representation of $\mathbb{R}$ in the unitary group of $M$ and there exists a positive self-adjoint operator $A$ affiliated with $M$ such that

$$
\alpha\left(\Psi\left(.,-\gamma_{t}\right)\right)=A^{i t}, \quad \text { for all } t \in \mathbb{R} .
$$

We can also define a positive self-adjoint operator $B$ affiliated with $M$ such that

$$
\alpha\left(\Psi\left(-\gamma_{t}, .\right)\right)=B^{i t} .
$$

We obtain :

$$
u_{t}=\lambda^{i \frac{t^{2}}{2}} A^{i t}, \quad v_{t}=\lambda^{i \frac{t^{2}}{2}} B^{i t} .
$$

Thus, we have $\varphi_{\Omega}=\varphi_{A}$ and $\psi_{\Omega}=\psi_{B}$, where $\varphi_{A}$ and $\psi_{B}$ are the weights defined by S. Vaes in [13].

One can also compute the dual $C^{*}$-algebra and the dual comultiplication. We put:

$$
L_{\gamma}=\alpha\left(u_{\gamma}\right), \quad R_{\gamma}=J L_{\gamma} J, \quad \text { for all } \gamma \in \hat{G} .
$$

From the representation $\gamma \mapsto L_{\gamma}$ we get the unital *-homomorphism $\lambda_{L}$ : $L^{\infty}(G) \rightarrow M$ and from the representation $\gamma \mapsto R_{\gamma}$ we get the unital normal *-homomorphism $\lambda_{R}: L^{\infty}(G) \rightarrow M^{\prime}$. Let $\hat{A}$ be the reduced $C^{*}$-algebra of $(M, \Delta)$. We can define an action of $\hat{G}^{2}$ on $\hat{A}$ by

$$
\alpha_{\gamma_{1}, \gamma_{2}}(x)=L_{\gamma_{1}} R_{\gamma_{2}} x R_{\gamma_{2}}^{*} L_{\gamma_{1}}^{*} .
$$

Let us consider the crossed product $C^{*}$-algebra $B=\hat{G}^{2}{ }_{\alpha} \ltimes \hat{A}$. We will denote by $\lambda$ the canonical morphism from $\hat{G}^{2}$ to the unitary group of $M(B)$ continuous in the strict topology on $\mathrm{M}(B), \pi \in \operatorname{Mor}(\hat{A}, B)$ the canonical morphism and $\theta$ the dual action of $G^{2}$ on $B$. Recall that the triplet $\left(\hat{G}^{2}, \lambda, \theta\right)$ is a $\hat{G}^{2}$-product. Let us denote by $\left(\hat{G}^{2}, \lambda, \theta^{\Psi}\right)$ the $\hat{G}^{2}$-product obtained by deformation of the $\hat{G}^{2}$-product $\left(\hat{G}^{2}, \lambda, \theta\right)$ by the bicharacter $\omega(g, h, s, t):=\overline{\Psi(g, s)} \Psi(h, t)$ on $G^{2}$.

The dual deformed action $\theta^{\Psi}$ is done by

$$
\theta_{\left(g_{1}, g_{2}\right)}^{\Psi}(x)=U_{g_{1}} V_{g_{2}} \theta_{\left(g_{1}, g_{2}\right)}(x) U_{g_{1}}^{*} V_{g_{2}}^{*}, \quad \text { for any } g_{1}, g_{2} \in G, x \in B,
$$

where $U_{g}=\lambda_{L}\left(\Psi_{g}^{*}\right), V_{g}=\lambda_{R}\left(\Psi_{g}\right), \Psi_{g}(h)=\Psi(h, g)$.

Considering $\Psi_{g}$ as an element of $\hat{G}$, we get a morphism from $G$ to $\hat{G}$, also noted $\Psi$, such that $\Psi(g)=\Psi_{g}$. With these notations, one has $U_{g}=u_{(\Psi(-g), 0)}$ and $V_{g}=u_{(0, \Psi(g))}$. Then the action $\theta^{\Psi}$ on $\pi(\hat{A})$ is done by

$$
\theta_{\left(g_{1}, g_{2}\right)}^{\Psi}(\pi(x))=\pi\left(\alpha_{\left(\Psi\left(-g_{1}\right), \Psi\left(g_{2}\right)\right)}(x)\right) .
$$

Let us consider the Landstad algebra $A^{\Psi}$ associated with this $\hat{G}^{2}$-product. By definition of $\alpha$ and the universality of the crossed product we get a morphism

$$
\rho \in \operatorname{Mor}(B, \mathcal{K}(H)), \quad \rho\left(\lambda_{\gamma_{1}, \gamma_{2}}\right)=L_{\gamma_{1}} R_{\gamma_{2}} \quad \text { et } \quad \rho(\pi(x))=x .
$$


It is shown in [4] that $\rho\left(A^{\Psi}\right)=\hat{A}_{\Omega}$ and that $\rho$ is injective on $A^{\Psi}$. This gives a canonical isomorphism $A^{\Psi} \simeq \hat{A}_{\Omega}$. In the sequel we identify $A^{\Psi}$ with $\hat{A}_{\Omega}$. The comultiplication can be described in the following way. First, one can show that, using universality of the crossed product, there exists a unique morphism $\Gamma \in \operatorname{Mor}(B, B \otimes B)$ such that:

$$
\Gamma \circ \pi=(\pi \otimes \pi) \circ \hat{\Delta} \quad \text { and } \quad \Gamma\left(\lambda_{\gamma_{1}, \gamma_{2}}\right)=\lambda_{\gamma_{1}, 0} \otimes \lambda_{0, \gamma_{2}} .
$$

Then we introduce the unitary $\Upsilon=\left(\lambda_{R} \otimes \lambda_{L}\right)(\tilde{\Psi}) \in \mathrm{M}(B \otimes B)$, where $\tilde{\Psi}(g, h)=$ $\Psi(g, g h)$. This allows us to define the *-morphism $\Gamma_{\Omega}(x)=\Upsilon \Gamma(x) \Upsilon^{*}$ from $B$ to $\mathrm{M}(B \otimes B)$. One can show that $\Gamma_{\Omega} \in \operatorname{Mor}\left(A^{\Psi}, A^{\Psi} \otimes A^{\Psi}\right)$ is the comultiplication on $A^{\Psi}$.

Note that if $M=\mathcal{L}(G)$ and $K$ is an abelian closed subgroup of $G$, the action $\alpha$ of $K^{2}$ on $C_{0}(G)$ is the left-right action.

\section{$3 \quad$ Twisting of the group of $2 \times 2$ upper triangular matrices with determinant 1}

Consider the following subgroup of $S L_{2}(\mathbb{C})$ :

$$
G:=\left\{\left(\begin{array}{cc}
z & \omega \\
0 & z^{-1}
\end{array}\right), z \in \mathbb{C}^{*}, \omega \in \mathbb{C}\right\}
$$

Let $K \subset G$ be the subgroup of diagonal matrices in $G$, i.e. $K=\mathbb{C}^{*}$. The elements of $G$ will be denoted by $(z, \omega), z \in \mathbb{C}, \omega \in \mathbb{C}^{*}$. The modular function of $G$ is

$$
\delta_{G}((z, \omega))=|z|^{-2} .
$$

Thus, the morphism $\left(t \mapsto \gamma_{t}\right)$ from $\mathbb{R}$ to $\widehat{\mathbb{C}^{*}}$ is given by

$$
\left\langle\gamma_{t}, z\right\rangle=|z|^{2 i t}, \quad \text { for all } z \in \mathbb{C}^{*}, t \in \mathbb{R} .
$$

We can identify $\widehat{\mathbb{C}^{*}}$ with $\mathbb{Z} \times \mathbb{R}_{+}^{*}$ in the following way:

$$
\mathbb{Z} \times \mathbb{R}_{+}^{*} \rightarrow \widehat{\mathbb{C}^{*}}, \quad(n, \rho) \mapsto \gamma_{n, \rho}=\left(r e^{i \theta} \mapsto e^{i \ln r \ln \rho} e^{i n \theta}\right) .
$$

Under this identification, $\gamma_{t}$ is the element $\left(0, e^{t}\right)$ of $\mathbb{Z} \times \mathbb{R}_{+}^{*}$. For all $x \in \mathbb{R}$, we define a bicharacter on $\mathbb{Z} \times \mathbb{R}_{+}^{*}$ by

$$
\Psi_{x}((n, \rho),(k, r))=e^{i x(k \ln \rho-n \ln r)} .
$$

We denote by $\left(M_{x}, \Delta_{x}\right)$ the twisted l.c. quantum group. We have:

$$
\Psi_{x}\left((n, \rho), \gamma_{t}^{-1}\right)=e^{i x t n}=u_{e^{i x t}}((n, \rho)) .
$$

In this way we obtain the operator $A_{x}$ deforming the Plancherel weight:

$$
A_{x}^{i t}=\alpha\left(u_{e^{i x t}}\right)=\lambda_{\left(e^{i t x}, 0\right)}^{G} .
$$


In the same way we compute the operator $B_{x}$ deforming the Plancherel weight:

$$
B_{x}^{i t}=\lambda_{\left(e^{-i x t}, 0\right)}^{G}=A_{x}^{-i t} .
$$

Thus, we obtain for the modular element :

$$
\delta_{x}^{i t}=A_{x}^{-i t} B_{x}^{i t}=\lambda_{\left(e^{-2 i t x}, 0\right)}^{G} .
$$

The antipode is not deformed. The scaling group is trivial but, if $x \neq 0$, $\left(M_{x}, \Delta_{x}\right)$ is not a Kac algebra because $\delta_{x}$ is not affiliated with the center of $M$. Let us look if $\left(M_{x}, \Delta_{x}\right)$ can be isomorphic for different values of $x$. One can remark that, since $\Psi_{-x}=\Psi_{x}^{*}$ is antisymmetric and $\Delta$ is cocommutative, we have $\Delta_{-x}=\sigma \Delta_{x}$, where $\sigma$ is the flip on $\mathcal{L}(G) \otimes \mathcal{L}(G)$. Thus, $\left(M_{-x}, \Delta_{-x}\right) \simeq\left(M_{x}, \Delta_{x}\right)^{\text {op }}$, where "op" means the opposite quantum group. So, it suffices to treat only strictly positive values of $x$. The twisting deforms only the comultiplication, the weights and the modular element. The simplest invariant distinguishing the $\left(M_{x}, \Delta_{x}\right)$ is then the specter of the modular element. Using the Fourier transformation in the first variable, on has immediately $\operatorname{Sp}\left(\delta_{x}\right)=q_{x}^{\mathbb{Z}} \cup\{0\}$, where $q_{x}=e^{-2 x}$. Thus, if $x \neq y, x>0, y>0$, one has $q_{x}^{\mathbb{Z}} \neq q_{y}^{\mathbb{Z}}$ and, consequently, $\left(M_{x}, \Delta_{x}\right)$ and $\left(M_{y}, \Delta_{y}\right)$ are non isomorphic.

We compute now the dual $C^{*}$-algebra. The action of $K^{2}$ on $C_{0}(G)$ can be lifted to its Lie algebra $\mathbb{C}^{2}$. The lifting does not change the result of the deformation (see [5], Proposition 3.17) but simplify calculations. The action of $\mathbb{C}^{2}$ on $C_{0}(G)$ will be denoted by $\rho$. One has

$$
\rho_{z_{1}, z_{2}}(f)(z, \omega)=f\left(e^{z_{2}-z_{1}} z, e^{-\left(z_{1}+z_{2}\right)} \omega\right) .
$$

The group $\mathbb{C}$ is self-dual, the duality is given by

$$
\left(z_{1}, z_{2}\right) \mapsto \exp \left(i \operatorname{Im}\left(z_{1} z_{2}\right)\right) .
$$

The generators $u_{z}, z \in \mathbb{C}$, of $C_{0}(\mathbb{C})$ are given by

$$
u_{z}(w)=\exp (i \operatorname{Im}(z w)), \quad z, w \in \mathbb{C} .
$$

Let $x \in \mathbb{R}$. We will consider the following bicharacter on $\mathbb{C}$ :

$$
\Psi_{x}\left(z_{1}, z_{2}\right)=\exp \left(i x \operatorname{Im}\left(z_{1} \bar{z}_{2}\right)\right) .
$$

Let $B$ be the crossed product $C^{*}$-algebra $\mathbb{C}^{2} \ltimes C_{0}(G)$. We denote by $\left(\left(z_{1}, z_{2}\right) \mapsto\right.$ $\left.\lambda_{z_{1}, z_{2}}\right)$ the canonical group homomorphism from $G$ to the unitary group of $\mathrm{M}(B)$, continuous for the strict topology, and $\pi \in \operatorname{Mor}\left(C_{0}(G), B\right)$ the canonical homomorphism. Also we denote by $\lambda \in \operatorname{Mor}\left(C_{0}\left(G^{2}\right), B\right)$ the morphism given by the representation $\left(\left(z_{1}, z_{2}\right) \mapsto \lambda_{z_{1}, z_{2}}\right)$. Let $\theta$ be the dual action of $\mathbb{C}^{2}$ on $B$. We have, for all $z, w \in \mathbb{C}, \Psi_{x}(w, z)=u_{x}(w)$. The deformed dual action is given by

$$
\theta_{z_{1}, z_{2}}^{\Psi_{x}}(b)=\lambda_{-x \bar{z}_{1}, x \bar{z}_{2}} \theta_{z_{1}, z_{2}}(b) \lambda_{-x \bar{z}_{1}, x \bar{z}_{2}}^{*} .
$$

Recall that

$$
\theta_{z_{1}, z_{2}}^{\Psi_{x}}(\lambda(f))=\theta_{z_{1}, z_{2}}(\lambda(f))=\lambda\left(f\left(\cdot-z_{1}, \cdot-z_{2}\right)\right), \quad \forall f \in C_{b}\left(\mathbb{C}^{2}\right) .
$$


Let $\hat{A}_{x}$ be the associated Landstad algebra. We identify $\hat{A}_{x}$ with the reduced $C^{*}$-algebra of $\left(M_{x}, \Delta_{x}\right)$. We will now construct two normal operators affiliated with $\hat{A}_{x}$, which generate $\hat{A}_{x}$. Let $a$ and $b$ be the coordinate functions on $G$, and $\alpha=\pi(a), \beta=\pi(b)$. Then $\alpha$ and $\beta$ are normal operators, affiliated with $B$, and one can see, using (5), that

$$
\lambda_{z_{1}, z_{2}} \alpha \lambda_{z_{1}, z_{2}}^{*}=e^{z_{2}-z_{1}} \alpha, \quad \lambda_{z_{1}, z_{2}} \beta \lambda_{z_{1}, z_{2}}^{*}=e^{-\left(z_{1}+z_{2}\right)} \beta .
$$

We can deduce, using (6), that

$$
\theta_{z_{1}, z_{2}}^{\Psi_{x}}(\alpha)=e^{x\left(\bar{z}_{1}+\bar{z}_{2}\right)} \alpha, \quad \theta_{z_{1}, z_{2}}^{\Psi_{x}}(\beta)=e^{x\left(\bar{z}_{1}-\bar{z}_{2}\right)} \beta .
$$

Let $T_{l}$ and $T_{r}$ be the infinitesimal generators of the left and right shift respectively, i.e. $T_{l}$ and $T_{r}$ are normal, affiliated with $B$, and

$$
\lambda_{z_{1}, z_{2}}=\exp \left(i \operatorname{Im}\left(z_{1} T_{l}\right)\right) \exp \left(i \operatorname{Im}\left(z_{2} T_{r}\right)\right), \quad \text { for all } z_{1}, z_{2} \in \mathbb{C} .
$$

Thus, we have:

$$
\lambda(f)=f\left(T_{l}, T_{r}\right), \quad \text { for all } f \in C_{b}\left(\mathbb{C}^{2}\right) .
$$

Let $U=\lambda\left(\Psi_{x}\right)$, we define the following normal operators affiliated with $B$ :

$$
\hat{\alpha}=U^{*} \alpha U, \hat{\beta}=U \beta U^{*} .
$$

Proposition 1 The operators $\hat{\alpha}$ and $\hat{\beta}$ are affiliated with $\hat{A}_{x}$ and generate $\hat{A}_{x}$.

Proof. First let us show that $f(\hat{\alpha}), f(\hat{\beta}) \in \mathrm{M}\left(\hat{A}_{t}\right)$, for all $f \in C_{0}(\mathbb{C})$. One has, using (7):

$$
\begin{aligned}
\theta_{z_{1}, z_{2}}^{\Psi_{x}}(U) & =\lambda\left(\Psi_{x}\left(.-z_{1}, .-z_{2}\right)\right) \\
& =U e^{i x \operatorname{Im}\left(-\bar{z}_{2} T_{l}\right)} e^{i x \operatorname{Im}\left(\bar{z}_{1} T_{r}\right)} \Psi_{x}\left(z_{1}, z_{2}\right) \\
& =U \lambda_{-x \bar{z}_{2}, x \bar{z}_{1}} \Psi_{x}\left(z_{1}, z_{2}\right) .
\end{aligned}
$$

Now, using (9) and (8), we obtain:

$$
\theta_{z_{1}, z_{2}}^{\Psi_{x}}(\hat{\alpha})=\hat{\alpha}, \quad \theta_{z_{1}, z_{2}}^{\Psi_{x}}(\hat{\beta})=\hat{\beta}, \quad \text { for all } z_{1}, z_{2} \in \mathbb{C} .
$$

Thus, for all $f \in C_{0}(\mathbb{C}), f(\hat{\alpha})$ and $f(\hat{\beta})$ are fixed points for the action $\theta^{\Psi_{x}}$. Let $f \in C_{0}(\mathbb{C})$. Using (8) we find:

$$
\begin{aligned}
& \lambda_{z_{1}, z_{2}} f(\hat{\alpha}) \lambda_{z_{1}, z_{2}}^{*}=U^{*} f\left(e^{z_{2}-z_{1}} \alpha\right) U \\
& \lambda_{z_{1}, z_{2}} f(\hat{\beta}) \lambda_{z_{1}, z_{2}}^{*}=U^{*} f\left(e^{-\left(z_{1}+z_{2}\right)} \beta\right) U .
\end{aligned}
$$

Because $f$ is continuous and vanish at infinity, the applications

$$
\left(z_{1}, z_{2}\right) \mapsto \lambda_{z_{1}, z_{2}} f(\hat{\alpha}) \lambda_{z_{1}, z_{2}}^{*} \quad \text { and } \quad\left(z_{1}, z_{2}\right) \mapsto \lambda_{z_{1}, z_{2}} f(\hat{\beta}) \lambda_{z_{1}, z_{2}}^{*}
$$

are norm-continuous and $f(\hat{\alpha}), f(\hat{\beta}) \in M\left(\hat{A}_{x}\right)$, for all $f \in C_{0}(\mathbb{C})$. 
Taking in mind Proposition 4 (see Appendix), in order to show that $\hat{\alpha}$ is affiliated with $\hat{A}_{x}$, it suffices to show that the vector space $\mathcal{I}$ generated by $f(\hat{\alpha}) a$, with $f \in C_{0}(\mathbb{C})$ and $a \in \hat{A}_{x}$, is dense in $\hat{A}_{x}$. Using (10), we see that $\mathcal{I}$ is globally invariant under the action implemented by $\lambda$. Let $g(z)=$ $(1+\bar{z} z)^{-1}$. As $\lambda\left(C_{0}\left(\mathbb{C}^{2}\right)\right) U=\lambda\left(C_{0}\left(\mathbb{C}^{2}\right)\right)$, we can deduce that the closure of $\lambda\left(C_{0}\left(\mathbb{C}^{2}\right)\right) g(\hat{\alpha}) \hat{A}_{x} \lambda\left(C_{0}\left(\mathbb{C}^{2}\right)\right)$ is equal to

$$
\left[\lambda\left(C_{0}\left(\mathbb{C}^{2}\right)\right)\left(1+\alpha^{*} \alpha\right)^{-1} U^{*} \hat{A}_{x} \lambda\left(C_{0}\left(\mathbb{C}^{2}\right)\right)\right] .
$$

As the set $U^{*} \hat{A}_{x} \lambda\left(C_{0}\left(\mathbb{C}^{2}\right)\right)$ is dense in $B$ and $\alpha$ is affiliated with $B$, the set $\lambda\left(C_{0}\left(\mathbb{C}^{2}\right)\right)\left(1+\alpha^{*} \alpha\right)^{-1} U^{*} \hat{A}_{x} \lambda\left(C_{0}\left(\mathbb{C}^{2}\right)\right)$ is dense in $B$. Moreover, it is included in $\lambda\left(C_{0}\left(\mathbb{C}^{2}\right)\right) \mathcal{I} \lambda\left(C_{0}\left(\mathbb{C}^{2}\right)\right)$, so $\lambda\left(C_{0}\left(\mathbb{C}^{2}\right)\right) \mathcal{I} \lambda\left(C_{0}\left(\mathbb{C}^{2}\right)\right)$ is dense in $B$. We conclude, using Lemma 1 , that $\mathcal{I}$ is dense in $\hat{A}_{x}$. One can show in the same way that $\hat{\beta}$ is affiliated with $\hat{A}_{x}$.

Now, let us show that $\hat{\alpha}$ and $\hat{\beta}$ generate $\hat{A}_{x}$. By Proposition 5 it suffices to show that

$$
\mathcal{V}=\left\langle f(\hat{\alpha}) g(\hat{\beta}), f, g \in C_{0}(\mathbb{C})\right\rangle
$$

is a dense vector subspace of $\hat{A}_{x}$. We have shown above that the elements of $\mathcal{V}$ satisfy the two first Landstad's conditions. Let

$$
\mathcal{W}=\left[\lambda\left(C_{0}\left(\mathbb{C}^{2}\right)\right) \mathcal{V} \lambda\left(C_{0}\left(\mathbb{C}^{2}\right)\right)\right] .
$$

We will show that $\mathcal{W}=B$. This proves that the elements of $\mathcal{V}$ satisfy the third Landstad's condition, and then $\mathcal{V} \subset \hat{A}_{x}$. Then (10) shows that $\mathcal{V}$ is globally invariant under the action implemented by $\lambda$, so $\mathcal{V}$ is dense in $\hat{A}_{x}$ by Lemma 1 One has:

$$
\mathcal{W}=\left[x U^{*} f(\alpha) U^{2} g(\beta) U^{*} y, f, g \in C_{0}(\mathbb{C}), x, y \in \lambda\left(C_{0}\left(\mathbb{C}^{2}\right)\right)\right] .
$$

Because $U$ is unitary, we can substitute $x$ with $x U$ and $y$ with $U y$ without changing $\mathcal{W}$ :

$$
\mathcal{W}=\left[x f(\alpha) U^{2} g(\beta) y, f, g \in C_{0}(\mathbb{C}), x, y \in \lambda\left(C_{0}\left(\mathbb{C}^{2}\right)\right)\right] .
$$

Using, for all $f \in C_{0}(\mathbb{C})$, the norm-continuity of the application

$$
\left(z_{1}, z_{2}\right) \mapsto \lambda_{z_{1}, z_{2}} f(\alpha) \lambda_{z_{1}, z_{2}}^{*}=e^{z_{2}-z_{1}} \alpha
$$

one deduces that

$$
\begin{aligned}
& {\left[f(\alpha) x, f \in C_{0}(\mathbb{C}), x \in \lambda\left(C_{0}\left(\mathbb{C}^{2}\right)\right)\right]} \\
& =\left[x f(\alpha), f \in C_{0}(\mathbb{C}), x \in \lambda\left(C_{0}\left(\mathbb{C}^{2}\right)\right)\right] .
\end{aligned}
$$

In particular,

$$
\mathcal{W}=\left[f(\alpha) x U^{2} g(\beta) y, f, g \in C_{0}(\mathbb{C}), x, y \in \lambda\left(C_{0}\left(\mathbb{C}^{2}\right)\right)\right] .
$$


Now we can commute $g(\beta)$ and $y$, and we obtain:

$$
\mathcal{W}=\left[f(\alpha) x U^{2} y g(\beta), f, g \in C_{0}(\mathbb{C}), x, y \in \lambda\left(C_{0}\left(\mathbb{C}^{2}\right)\right)\right] .
$$

Substituting $x \mapsto x U^{*}, y \mapsto U^{*} y$, one has:

$$
\mathcal{W}=\left[f(\alpha) x y g(\beta), f, g \in C_{0}(\mathbb{C}), x, y \in \lambda\left(C_{0}\left(\mathbb{C}^{2}\right)\right)\right] .
$$

Commuting back $f(\alpha)$ with $x$ and $g(\beta)$ with $y$, we obtain:

$$
\mathcal{W}=\left[x f(\alpha) g(\beta) y, f, g \in C_{0}(\mathbb{C}), x, y \in \lambda\left(C_{0}\left(\mathbb{C}^{2}\right)\right)\right]=B .
$$

This concludes the proof.

We will now find the commutation relations between $\hat{\alpha}$ and $\hat{\beta}$.

Proposition 2 One has:

1. $\alpha$ et $T_{l}^{*}+T_{r}^{*}$ strongly commute and $\hat{\alpha}=e^{x\left(T_{l}^{*}+T_{r}^{*}\right)} \alpha$.

2. $\beta$ et $T_{l}^{*}-T_{r}^{*}$ strongly commute and $\hat{\beta}=e^{x\left(T_{l}^{*}-T_{r}^{*}\right)} \beta$.

Thus, the polar decompositions are given by:

$$
\begin{array}{ll}
\operatorname{Ph}(\hat{\alpha})=e^{-i x \operatorname{Im}\left(T_{l}+T_{r}\right)} \operatorname{Ph}(\alpha), & |\hat{\alpha}|=e^{x \operatorname{Re}\left(T_{l}+T_{r}\right)}|\alpha|, \\
\operatorname{Ph}(\hat{\beta})=e^{-i x \operatorname{Im}\left(T_{l}-T_{r}\right)} \operatorname{Ph}(\beta), & |\hat{\beta}|=e^{x \operatorname{Re}\left(T_{l}-T_{r}\right)}|\beta| .
\end{array}
$$

Moreover, we have the following relations:

1. $|\hat{\alpha}|$ and $|\hat{\beta}|$ strongly commute,

2. $\operatorname{Ph}(\hat{\alpha}) \operatorname{Ph}(\hat{\beta})=\operatorname{Ph}(\hat{\beta}) \operatorname{Ph}(\hat{\alpha})$,

3. $\operatorname{Ph}(\hat{\alpha})|\hat{\beta}| P h(\hat{\alpha})^{*}=e^{4 x}|\hat{\beta}|$,

4. $\operatorname{Ph}(\hat{\beta})|\hat{\alpha}| \operatorname{Ph}(\hat{\beta})^{*}=e^{4 x}|\hat{\alpha}|$.

Proof. Using (8), we find, for all $z \in \mathbb{C}$ :

$$
e^{i \operatorname{Im}\left(z\left(T_{l}^{*}+T_{r}^{*}\right)\right)} \alpha e^{-i \operatorname{Im}\left(z\left(T_{l}^{*}+T_{r}^{*}\right)\right)}=\lambda_{-\bar{z},-\bar{z}} \alpha \lambda_{-\bar{z},-\bar{z}}^{*}=e^{-\bar{z}+\bar{z}} \alpha=\alpha .
$$

Thus, $T_{l}^{*}+T_{r}^{*}$ and $\alpha$ strongly commute. Moreover, because $e^{i x \operatorname{Im} T_{l} T_{l}^{*}}=1$, one has:

$$
\hat{\alpha}=e^{-i x \operatorname{Im} T_{l} T_{r}^{*}} \alpha e^{i x \operatorname{Im} T_{l} T_{r}^{*}}=e^{-i x \operatorname{Im} T_{l}\left(T_{l}+T_{r}\right)^{*}} \alpha e^{i x \operatorname{Im} T_{l}\left(T_{l}+T_{r}\right)^{*}} .
$$

We can now prove the point 1 using the equality $e^{-i x \operatorname{Im} T_{l} \omega} \alpha e^{i x \operatorname{Im} T_{l} \omega}=e^{x \omega} \alpha$, the preceding equation and the fact that $T_{l}^{*}+T_{r}^{*}$ and $\alpha$ strongly commute. The proof of the second assertion is similar and the polar decompositions follows. From (8) we deduce : 


$$
\begin{aligned}
e^{-i x \operatorname{Im}\left(T_{r}-T_{l}\right)} \alpha e^{i x \operatorname{Im}\left(T_{r}-T_{l}\right)} & =e^{-2 x} \alpha, \\
e^{i x \operatorname{Im}\left(T_{l}+T_{r}\right)} \beta e^{-i x \operatorname{Im}\left(T_{l}+T_{r}\right)} & =e^{-2 x} \beta, \\
e^{i x \operatorname{Re}\left(T_{r}-T_{l}\right)} \alpha e^{-i x \operatorname{Re}\left(T_{r}-T_{l}\right)} & =e^{2 i x} \alpha, \\
e^{i x \operatorname{Re}\left(T_{l}+T_{r}\right)} \beta e^{-i x \operatorname{Re}\left(T_{l}+T_{r}\right)} & =e^{-2 i x} \beta .
\end{aligned}
$$

It is now easy to prove the last relations from the preceding equations and the polar decompositions.

We can now give a formula for the comultiplication.

Proposition 3 Let $\hat{\Delta}_{x}$ be the comultiplication on $\hat{A}_{x}$. One has:

$$
\hat{\Delta}_{x}(\hat{\alpha})=\hat{\alpha} \otimes \hat{\alpha}, \hat{\Delta}_{x}(\hat{\beta})=\hat{\alpha} \otimes \hat{\beta} \dot{+} \hat{\beta} \otimes \hat{\alpha}^{-1} .
$$

Proof. Using the Preliminaries, we have that $\hat{\Delta}_{x}=\Upsilon \Gamma(.) \Upsilon^{*}$, where

$$
\Upsilon=e^{i x \operatorname{Im} T_{r} \otimes T_{l}^{*}}
$$

and $\Gamma$ is given by

- $\Gamma\left(T_{l}\right)=T_{l} \otimes 1, \Gamma\left(T_{r}\right)=1 \otimes T_{r} ;$

- $\Gamma$ restricted to $C_{0}(G)$ is equal to the comultiplication $\Delta_{G}$.

Define $R=\Upsilon \Gamma\left(U^{*}\right)$. One has $\Delta_{x}(\hat{\alpha})=R(\alpha \otimes \alpha) R^{*}$. Thus, it is sufficient to show that $(U \otimes U) R$ commute with $\alpha \otimes \alpha$. Indeed, in this case, one has

$\hat{\Delta}_{x}(\hat{\alpha})=R(\alpha \otimes \alpha) R^{*}=\left(U^{*} \otimes U^{*}\right)(U \otimes U) R(\alpha \otimes \alpha) R^{*}\left(U^{*} \otimes U^{*}\right)(U \otimes U)=\hat{\alpha} \otimes \hat{\alpha}$.

Let us show that $(U \otimes U) R$ commute with $\alpha \otimes \alpha$. From the equality $U=$ $e^{i x \operatorname{Im} T_{l} T_{r}^{*}}$, we deduce that

$$
\Gamma\left(U^{*}\right)=e^{-i x \operatorname{Im} T_{l} \otimes T_{r}^{*}}, \quad U \otimes U=e^{i x \operatorname{Im}\left(T_{l} T_{r}^{*} \otimes 1+1 \otimes T_{l} T_{r}^{*}\right)} .
$$

Thus, $R=e^{-i x \operatorname{Im}\left(T_{r}^{*} \otimes T_{l}+T_{l} \otimes T_{r}^{*}\right)}$ and

$$
(U \otimes U) R=e^{i x \operatorname{Im}\left(T_{l} T_{r}^{*} \otimes 1+1 \otimes T_{l} T_{r}^{*}-T_{r}^{*} \otimes T_{l}-T_{l} \otimes T_{r}^{*}\right)} .
$$

Notice that

$T_{l} T_{r}^{*} \otimes 1+1 \otimes T_{l} T_{r}^{*}-T_{r}^{*} \otimes T_{l}-T_{l} \otimes T_{r}^{*}=\left(T_{l} \otimes 1-1 \otimes T_{l}\right)\left(T_{r}^{*} \otimes 1-1 \otimes T_{r}^{*}\right)$.

Thus, it suffices to show that $T_{l} \otimes 1-1 \otimes T_{l}$ and $T_{r}^{*} \otimes 1-1 \otimes T_{r}^{*}$ strongly commute with $\alpha \otimes \alpha$. This follows from the equations

$$
\begin{aligned}
& e^{i \operatorname{Im} z\left(T_{r}^{*} \otimes 1-1 \otimes T_{r}^{*}\right)}(\alpha \otimes \alpha) e^{-i \operatorname{Im} z\left(T_{r}^{*} \otimes 1-1 \otimes T_{r}^{*}\right)} \\
& =\left(\lambda_{0,-\bar{z}} \otimes \lambda_{0, \bar{z}}\right)(\alpha \otimes \alpha)\left(\lambda_{0,-\bar{z}} \otimes \lambda_{0, \bar{z}}\right)^{*} \\
& =e^{-\bar{z}} e^{\bar{z}} \alpha \otimes \alpha=\alpha \otimes \alpha, \quad \forall z \in \mathbb{C}
\end{aligned}
$$


and

$$
\begin{aligned}
& e^{i \operatorname{Im} z\left(T_{l} \otimes 1-1 \otimes T_{l}\right)}(\alpha \otimes \alpha) e^{-i \operatorname{Im} z\left(T_{l} \otimes 1-1 \otimes T_{l}\right)} \\
& =\left(\lambda_{z, 0} \otimes \lambda_{-z, 0}\right)(\alpha \otimes \alpha)\left(\lambda_{z, 0} \otimes \lambda_{-z, 0}\right)^{*} \\
& =e^{-z} e^{z} \alpha \otimes \alpha=\alpha \otimes \alpha, \quad \forall z \in \mathbb{C} .
\end{aligned}
$$

Put $S=\Upsilon \Gamma(U)$. One has:

$$
\hat{\Delta}_{x}(\hat{\beta})=S\left(\alpha \otimes \beta+\beta \otimes \alpha^{-1}\right) S^{*}=S(\alpha \otimes \beta) S^{*} \dot{+} S\left(\beta \otimes \alpha^{-1}\right) S^{*} .
$$

As before, we see that it suffices to show that $\left(U \otimes U^{*}\right) S$ commutes with $\alpha \otimes \beta$ and that $\left(U^{*} \otimes U\right) S$ commutes with $\beta \otimes \alpha^{-1}$, and one can check this in the same way .

Let us summarize the preceding results in the following corollary (see [16, 5] for the definition of commutation relation between unbounded operators):

Corollary 1 Let $q=e^{8 x}$. The $C^{*}$-algebra $\hat{A}_{x}$ is generated by 2 normal operators $\hat{\alpha}$ and $\hat{\beta}$ affiliated with $\hat{A}_{x}$ such that

$$
\hat{\alpha} \hat{\beta}=\hat{\beta} \hat{\alpha} \quad \hat{\alpha} \hat{\beta}^{*}=q \hat{\beta}^{*} \hat{\alpha} .
$$

Moreover, the comultiplication $\hat{\Delta}_{x}$ is given by

$$
\hat{\Delta}_{x}(\hat{\alpha})=\hat{\alpha} \otimes \hat{\alpha}, \hat{\Delta}_{x}(\hat{\beta})=\hat{\alpha} \otimes \hat{\beta} \dot{+} \hat{\beta} \otimes \hat{\alpha}^{-1} .
$$

Remark. One can show, using the results of [4], that the application $(q \mapsto$ $W_{q}$ ) which maps the parameter $q$ to the multiplicative unitary of the twisted l.c. quantum group is continuous in the $\sigma$-weak topology.

\section{Appendix}

Let us cite some results on operators affiliated with a $C^{*}$-algebra.

Proposition 4 Let $A \subset \mathcal{B}(H)$ be a non degenerated $C^{*}$-subalgebra and $T$ a normal densely defined closed operator on $H$. Let $\mathcal{I}$ be the vector space generated by $f(T) a$, where $f \in C_{0}(\mathbb{C})$ and $a \in A$. Then:

$$
(T \eta A) \Leftrightarrow\left(\begin{array}{c}
f(T) \in M(A) \text { for any } f \in C_{0}(\mathbb{C}) \\
\text { et } \mathcal{I} \text { is dense in } A
\end{array}\right) .
$$

Proof. If $T$ is affiliated with $A$, then it is clear that $f(T) \in \mathrm{M}(A)$ for any $f \in$ $C_{0}(\mathbb{C})$, and that $\mathcal{I}$ is dense in $A$ (because $\mathcal{I}$ contains $\left.\left(1+T^{*} T\right)^{-\frac{1}{2}} A\right)$. To show the converse, consider the ${ }^{*}$-homomorphism $\pi_{T}: C_{0}(\mathbb{C}) \rightarrow \mathrm{M}(A)$ given by $\pi_{T}(f)=$ $f(T)$. By hypothesis, $\pi_{T}\left(C_{0}(\mathbb{C})\right) A$ is dense in $A$. So, $\pi_{T} \in \operatorname{Mor}\left(C_{0}(\mathbb{C}), A\right)$ and $T=\pi_{T}(z \mapsto z)$ is then affiliated with $A$. 
Proposition 5 Let $A \subset \mathcal{B}(H)$ be a non degenerated $C^{*}$-subalgebra and $T_{1}, T_{2}, \ldots, T_{N}$ normal operators affiliated with $A$. Let us denote by $\mathcal{V}$ the vector space generated by the products of the form $f_{1}\left(T_{1}\right) f_{2}\left(T_{2}\right) \ldots f_{N}\left(T_{N}\right)$, with $f_{i} \in C_{0}(\mathbb{C})$. If $\mathcal{V}$ is a dense vector subspace of $A$, then $A$ is generated by $T_{1}, T_{2}, \ldots, T_{N}$.

Proof. This follows from Theorem 3.3 in [15].

\section{References}

[1] J. Bichon, J., A. De Rijdt, and S. Vaes, Ergodic coactions with large multiplicity and monoidal equivalence of quantum groups, Commun. Math. Phys., 22, 703-728, 2006

[2] M. Enock and J.-M. Schwartz, Kac algebras and duality of locally compact groups, Springer, Berlin, 1992.

[3] M. Enock and L. Vainerman, Deformation of a Kac algebra by an abelian subgroup, Commun. Math. Phys., 178, No. 3, 571-596, 1996

[4] P. Fima and L. Vainerman, Twisting of locally compact quantum groups. Deformation of the Haar measure., preprint.

[5] P. Kasprzak, Deformation of $C^{*}$-algebras by an action of abelian groups with dual 2-cocycle and Quantum Groups, preprint : arXiv:math.OA/0606333

[6] J. Kustermans and S. Vaes, Locally compact quantum groups, Ann. Sci. Ec. Norm. Super., IV, Ser. 33, No. 6, 547-934, 2000

[7] J. Kustermans and S. Vaes, Locally compact quantum groups in the von Neumann algebraic setting, Math. Scand., 92 (1), 68-92, 2003

[8] M. Landstad, Quantization arising from abelian subgroups, Int. J. Math., $5,897-936,1994$

[9] G.K. Pedersen, $C^{*}$-algebras and their automorphism groups, Academic Press, 1979.

[10] M. Rieffel, Deformation quantization for actions of $\mathbb{R}^{d}$, Memoirs A.M.S., 506,1993

[11] M. Takesaki and N. Tatsuuma, Duality and subgroups, Ann. of Math., 93, 344-364, 1971

[12] S. Stratila, Modular Theory in Operator Algebras, Abacus Press, Turnbridge Wells, England, 1981.

[13] S. Vaes, A Radon-Nikodym theorem for von Neuman algebras, J. Operator. Theory., 46, No.3, 477-489, 2001 
[14] L. Vainerman, 2-cocycles and twisting of Kac algebras, Commun. Math. Phys., 191, No. 3, 697-721, 1998

[15] S. L. Woronowicz, $C^{*}$-algebras generated by unbounded elements, Reviews in Math. Physics, 7, No. 3, 481-521, 1995

[16] S. L. Woronowicz, Quantum E(2) group and its Pontryagin dual, Lett. Math. Phys., 23, 251-263, 1991 